Bundesgesundheitsbl $2012 \cdot 55: 431-435$

DOI 10.1007/s00103-012-1443-1

Online publiziert: 25. Februar 2012

(c) Springer-Verlag 2012

I.K. Wolf $\cdot$ H. Knopf $\cdot$ C. Scheidt-Nave $\cdot$ B.M. Kurth

Robert Koch-Institut FG 22, Berlin

\title{
Möglichkeiten und Grenzen retrospektiver Todesursachenre- cherchen im Rahmen bundesweiter epidemiologischer Studien
}

holte Einverständniserklärung durchzuführen, wenn es nicht mehr möglich ist, die Einverständniserklärung einzuholen, der Forschungszweck einem legitimierten Gemeinschaftsinteresse dient und die erforderlichen Datenschutzbedingungen eingehalten werden [5].

\section{Rahmenbedingungen nach Bundesländern}

Grundsätzlich sind drei Hauptschritte zu beachten:

1. Einholen der Stellungnahmen der Datenschutzbeauftragten,

2. Einholen der Stellungnahmen der obersten Landesgesundheitsbehörde (i. d. R. Landesgesundheitsministerien),

3. Beantragung der Datenübermittlung bei den Datenhaltern [Gesundheitsämter, Institut für Rechtsmedizin, Gemeinsames Krebsregister (GKR) etc.].

In - Tab. 1 sind die Zugangswege und -bedingungen zur Erfassung von Todesursachen nach Bundesländern dargestellt.

In nahezu allen Bundesländern (mit Ausnahme von Nordrhein-Westfalen) findet eine begründete Ausnahmeregelung zur Recherche von Todesursachen die Unterstützung der Landesbeauftragten für Datenschutz und Informationsfreiheit.

In vier Bundesländern (Bremen, Hamburg, Saarland, Sachsen) können die Anträge auf Datenübermittlung mit befürwortender Stellungnahme der Landesdatenschutzbeauftragten direkt bei den
Gesundheitsämtern bzw. beim Bremer Institut für Rechtsmedizin gestellt werden. In Bremen müssen jedoch zuvor die Sterbebuchnummern beim Standesamt eingeholt werden.

In zehn Bundesländern (Baden-Württemberg, Bayern, Brandenburg, Hessen, Mecklenburg-Vorpommern, Niedersachsen, Rheinland-Pfalz, Sachsen-Anhalt, Schleswig-Holstein, Thüringen) können mit datenschutzrechtlicher Unbedenklichkeitserklärung in einem zweiten Schritt die Zustimmungen der obersten Landesgesundheitsbehörden eingeholt werden. In Baden-Württemberg, Rheinland-Pfalz und Schleswig-Holstein ist dieser zweite Schritt jedoch keine zwingende Voraussetzung, sondern eine Empfehlung, die den Gesundheitsämtern Handlungssicherheit geben sollte.

Die Mehrheit dieser Bundesländer (Baden-Württemberg, Brandenburg, Mecklenburg-Vorpommern, Niedersachsen, Rheinland-Pfalz, Sachsen- Anhalt, Schleswig-Holstein, Thüringen) ermöglicht, in einem dritten Schritt eine direkte Ermittlung der Todesursachen bei den Gesundheitsämtern. In Hessen müssen zuvor bei den Standesämtern die Sterbebuchnummern eingeholt werden. Kompliziert gestaltet sich der Zugang zu den Todesursachen in einem Gesundheitsamt in Hessen. Dort genügen nicht die Zustimmung des Landesbeauftragten für den Datenschutz und der obersten Gesundheitsbehörde sowie das Einholen der Sterbebuchnummern bei den Standesämtern, sondern es muss darüber hinaus noch eine Zustimmung beim Deut- 
Tab. 1 Übersicht der Aufbewahrungsorte/-fristen von Todesbescheinigungen und der Zugangsbedingungen. Stand Mai 2011

\begin{tabular}{|c|c|c|c|}
\hline Bundesland & Aufbewahrungsorte & $\begin{array}{l}\text { Aufbewah- } \\
\text { rungsfrist }\end{array}$ & Zugangsbedingungen \\
\hline $\begin{array}{l}\text { Baden- } \\
\text { Württem- } \\
\text { berg }\end{array}$ & $\begin{array}{l}\text { GA des Sterbeortes } \\
\text { Ab } 1968 \text { EDV }\end{array}$ & 30 Jahre & $\begin{array}{l}\text { 1. Zustimmung des/r Landesdatenschutzbeauftragte/n } \\
\text { 2. Zustimmung des GM nicht zwingend notwendig, könnte aber GAs Handlungssicher- } \\
\text { heit geben } \\
\text { 3. Einholen der Sterbebuchnummern bei Standesämtern empfehlenswert }\end{array}$ \\
\hline Bayern & GA des Sterbeortes & 30 Jahre & $\begin{array}{l}\text { 1. Zustimmung des/r Landesdatenschutzbeauftragte/n } \\
\text { 2. Zustimmung des Regierungsbezirkes, in dem die meisten Probanden verstorben sind } \\
\text { 3. Der zuständige Regierungsbezirk wird vom GM bestimmt }\end{array}$ \\
\hline Berlin & $\begin{array}{l}\text { Amt für Statistik Berlin-Bran- } \\
\text { denburg } \\
\text { Alle TBs gehen an das GKR, } \\
\text { nur Krebsdaten werden dort } \\
\text { registriert }\end{array}$ & $\begin{array}{l}3 \text { Jahre beim } \\
\text { Amt für Stati- } \\
\text { stik, seit } 1999 \\
\text { beim Krebsre- } \\
\text { gister }\end{array}$ & $\begin{array}{l}\text { 1. Zustimmung des/r Landesdatenschutzbeauftragte/n } \\
\text { 2. Recherchen über das GKR unter besonderen datenschutzrechtlichen Bedingungen } \\
\text { möglich } \\
\text { 3. Recherchen über Krankenhäuser/Ärzte, die die TBs ausgestellt haben }\end{array}$ \\
\hline $\begin{array}{l}\text { Branden- } \\
\text { burg }\end{array}$ & GA des Sterbeortes & 30 Jahre & $\begin{array}{l}\text { 1. Zustimmung des/r Landesdatenschutzbeauftragte/n } \\
\text { 2. Zustimmung des GM }\end{array}$ \\
\hline Bremen & $\begin{array}{l}\text { Stadt Bremen: IRZ } \\
\text { Bremerhaven: Magistrat der } \\
\text { Stadtgemeinde Bremerhaven } \\
\text { Alle: BREMI }\end{array}$ & 30 Jahre & $\begin{array}{l}\text { 1. Zustimmung des/r Landesdatenschutzbeauftragte/n } \\
\text { 2. Beim Standesamt Sterbebuchnummern einholen } \\
\text { 3. Für den Bremer Mortalitätsindex (BREMI) gelten besondere Forschungsbedingungen }\end{array}$ \\
\hline Hamburg & $\begin{array}{l}\text { Alle TBs werden beim GA } \\
\text { Wandsbek aufbewahrt }\end{array}$ & $\begin{array}{l}\text { Mindestens } \\
25 \text { Jahre, maxi- } \\
\text { mal } 30 \text { Jahre }\end{array}$ & $\begin{array}{l}\text { 1. Zustimmung des/r Landesdatenschutzbeauftragte/n } \\
\text { 2. Zustimmung des GA Wandsbek }\end{array}$ \\
\hline Hessen & Bei GA des Sterbeortes & $\begin{array}{l}50 \text { Jahre, ab } \\
1970\end{array}$ & $\begin{array}{l}\text { 1. Zustimmung des/r Landesdatenschutzbeauftragte/n } \\
\text { 2. Zustimmung des GM } \\
\text { 3. Bei Standesämtern die Sterbebuchnummern einholen } \\
\text { 4. Ein GA fordert Einholen der Zustimmung des Deutschen Landkreistags }\end{array}$ \\
\hline $\begin{array}{l}\text { Mecklen- } \\
\text { burg-Vor- } \\
\text { pommern }\end{array}$ & GA des Sterbeortes & 30 Jahre & $\begin{array}{l}\text { 1. Zustimmung des/r Landesdatenschutzbeauftragte/n } \\
\text { 2. Zustimmung des GM }\end{array}$ \\
\hline $\begin{array}{l}\text { Niedersach- } \\
\text { sen }\end{array}$ & GA des Sterbeortes & 30 Jahre & $\begin{array}{l}\text { 1. Zustimmung des/r Landesdatenschutzbeauftragte/n } \\
\text { 2. Zustimmung des GM }\end{array}$ \\
\hline $\begin{array}{l}\text { Nordrhein- } \\
\text { Westfalen }\end{array}$ & GA des Sterbeortes & 10 Jahre & $\begin{array}{l}\text { 1. Zustimmung des/r Landesdatenschutzbeauftragte/n } \\
\text { 2. Zustimmung des GM } \\
\text { 3. TU-Recherchen ohne Einverständniserklärung und ärztliche Schweigepflichtentbin- } \\
\text { dung nicht möglich }\end{array}$ \\
\hline $\begin{array}{l}\text { Rheinland- } \\
\text { Pfalz }\end{array}$ & $\begin{array}{l}\text { GA des Sterbeortes } \\
\text { Ab } 2011 \text { Aufbau Mortalitäts- } \\
\text { register }\end{array}$ & $\begin{array}{l}\text { Mindestens } \\
5 \text { Jahre }\end{array}$ & $\begin{array}{l}\text { 1. Zustimmung des/r Landesdatenschutzbeauftragte/n } \\
\text { 2. Zustimmung des GM nicht zwingend notwendig, könnte aber GAs Handlungssicher- } \\
\text { heit geben }\end{array}$ \\
\hline Saarland & GA des Sterbeortes & $\begin{array}{l}\text { Unbefristete } \\
\text { Aufbewahrung }\end{array}$ & $\begin{array}{l}\text { 1. Zustimmung des/r Landesdatenschutzbeauftragte/n } \\
\text { 2. GAs entscheiden selbst, ob sie Auskünfte erteilen }\end{array}$ \\
\hline Sachsen & GA des Sterbeortes & 30 Jahre bei GA & $\begin{array}{l}\text { 1. Zustimmung des/r Landesdatenschutzbeauftragte/n } \\
\text { 2. GAs entscheiden selbst, ob sie Auskünfte erteilen }\end{array}$ \\
\hline $\begin{array}{l}\text { Sachsen- } \\
\text { Anhalt }\end{array}$ & GA des Sterbeortes & 30 Jahre & $\begin{array}{l}\text { 1. Zustimmung des/r Landesdatenschutzbeauftragte/n } \\
\text { 2. Zustimmung des Gesundheitsministeriums }\end{array}$ \\
\hline $\begin{array}{l}\text { Schleswig- } \\
\text { Holstein }\end{array}$ & GA des Wohnortes & 30 Jahre & $\begin{array}{l}\text { 1. Zustimmung des/r Landesdatenschutzbeauftragte/n } \\
\text { 2. Zustimmung des GM nicht zwingend notwendig, könnte aber GAs Handlungssicher- } \\
\text { heit geben }\end{array}$ \\
\hline Thüringen & GA des Sterbeortes & 30 Jahre & $\begin{array}{l}\text { 1. Zustimmung des/r Landesdatenschutzbeauftragte } / n \\
\text { 2. Zustimmung des GM }\end{array}$ \\
\hline
\end{tabular}

schen Landkreistag beantragt werden. In Bayern bedarf es zusätzlich der Zustimmung des Regierungsbezirks, in dessen Einzugsbereich die meisten Probanden verstorben sind.

- Abb. 1 liefert eine zusammenfassende Darstellung der oben beschriebenen
Abläufe in 14 Bundesländern (ohne Berlin und Nordrhein-Westfalen). Auf Berlin und Nordrhein-Westfalen, für die jeweils besondere Bedingungen zutreffen, wird gesondert eingegangen.

In Berlin ist eine Todesursachenrecherche über die Gesundheitsämter auf- grund kurzer Aufbewahrungsfristen der Todesbescheinigungen (wenige Wochen) und anschließender Weiterleitung an das Amt für Statistik Berlin-Brandenburg nicht möglich. Da Statistische Ämter besonderen Datenschutzregeln unterliegen, können von dort nur absolut anonymi- 
Bundesgesundheitsbl 2012·55:431-435 DOI 10.1007/s00103-012-1443-1

(c) Springer-Verlag 2012

\section{I.K. Wolf $\cdot$ H. Knopf $\cdot$ C. Scheidt-Nave $\cdot$ B.M. Kurth}

\section{Möglichkeiten und Grenzen retrospektiver Todesursachenrecherchen im Rahmen bundesweiter epidemiologischer Studien}

\section{Zusammenfassung}

Die ursachenspezifische Mortalität bildet in longitudinal angelegten epidemiologischen Studien einen wichtigen Endpunkt, um kausale gesundheitliche Zusammenhänge zu erforschen und individuelle Verlaufsanalysen durchzuführen. Da in Deutschland kein nationales Mortalitätsregister existiert, werden länderspezifische datenschutzrechtliche Rahmenbedingungen und Zugangswege für die Todesursachenrecherche im Rahmen eines Pilotprojekts recherchiert und erprobt. Die Datengrundlage liefert eine Stichprobe verstorbener StudienteilnehmerInnen des Bundes-Gesundheitssurveys 1998. Unter den gegebenen Bedingungen ist die Recherche von Todesursachen in bundesweiten epidemiologischen Studien grundsätzlich möglich, allerdings mit einem hohen zeitlichen und personellen Aufwand verbunden. In Rheinland-
Pfalz muss zeitnah recherchiert werden, da die Aufbewahrungsfrist für Todesbescheinigungen bei den Gesundheitsämtern auf eine Mindestfrist von fünf Jahren begrenzt ist. In Berlin gestaltet sich die Forschungssituation besonders schwierig, da die Todesbescheinigungen zwar beim Amt für Statistik drei Jahre lang aufbewahrt werden, aber das Amt für Statistik keine pseudonymisierten Daten herausgeben darf. In Berlin können über die Ärzte und Krankenhäuser, die die Todesbescheinigungen ausgestellt haben, und über das GKR Berlin-Brandenburg (unter Einhaltung der besonderen datenschutzrechtlichen Bedingungen des Krebsregistergesetzes) für einen Teil der Verstorbenen die Todesursachen recherchiert werden. In Nordrhein-Westfalen wird grundsätzlich die zu Lebzeiten eingeholte schriftliche Einwilligung zur Recherche der To- desursache unter explizitem Einschluss einer ärztlichen Schweigepflichtentbindung vorausgesetzt. In allen anderen Bundesländern sind Ausnahmeregelungen möglich, die in jedem Fall der Zustimmung des Landesdatenschutzbeauftragten bedürfen. Die Ergebnisse des Pilotprojekts unterstreichen die Forderung nach einem nationalen Mortalitätsregister. Bis zu dessen Etablierung könnte die pilotierte Vorgehensweise genutzt und in Zusammenarbeit zwischen Bundes- und Landesbeauftragten für Datenschutz sowie den obersten Landesgesundheitsbehörden weiter optimiert werden, um Datenverluste zu vermeiden und Ressourcen effizient einzusetzen.

Schlüsselwörter

Todesursachen - Todesursachenrecherche . Epidemiologische Studien · Deutschland

\section{Possibilites and limitations of retrospective research on cause of death within the framework of a nationwide epidemiological study}

\section{Abstract}

Cause-specific mortality is an important endpoint in longitudinal epidemiological studies to research causal health links and carry out individual process analyses. As in Germany no national mortality register exists, state-specific data-protection conditions for and approaches to cause of death studies were researched and tested within the framework of a pilot project. The database was provided by a sample of deceased study participants from the 1998 nationwide health survey. Under the given conditions, cause of death research in a nationwide epidemiological study is possible in principle, but requires a great deal of time and effort. In Rhineland-Palatinate research needs to be carried out in a timely manner as the health authorities' retention period for death certificates is limited to minimum of 5 years. In Berlin the research situation is particularly difficult as, while the death certificates are held 3 years by the statistics bureau, this department cannot release any pseudonymous data. In Berlin it is possible to research the cause of death for some of the deceased through the doctors and hospitals that issued the death certificates and through the GKR Berlin-Brandenburg (in keeping with the special data protection measures of the cancer registry). In North Rhine Westphalia the written consent obtained during people's lifetimes, including an explicit release from medical confidentiality, is required to carry out cause of death re- search. In all other German states exceptions are possible, in all cases requiring the consent of the state's data protection commissioners. The results of the pilot project underline the need for a national mortality register. Until this is established the approach used in the pilot study can be used and, working together with the state and national data protection authorities and with the highest national health authorities, can be further optimized in order to avoid losing data and to use resources efficiently.

Keywords

Cause of death . Cause of death research . Epidemiological studies · Germany sierte, aber nicht für die Recherchen benötigte pseudonymisierte Daten übermittelt werden. Unter Beachtung der besonderen datenschutzrechtlichen Auflagen (geregelt im Gesetz über das Krebsregister - KRG), ist es möglich, zumindest für einen Teil der Verstorbenen aus Berlin die Todesursachen über das GKR zu recherchieren. Für die verbleibenden Sterbefälle mit ungeklärten Todesursachen werden über das Amt für Statistik und die
Bezirksämter die Krankenhäuser/Ärzte ermittelt, die die Todesbescheinigungen ausgestellt haben. Nach Kontaktieren dieser Krankenhäuser/Ärzte können in einigen Fällen die Todesursachen möglicherweise noch recherchiert werden.

In Nordrhein-Westfalen kann keine Ausnahmeregelung zur Todesursachenrecherche erworben werden. Die Unbedenklichkeitserklärung des Landesdatenschutzbeauftragten ist hier grundsätzlich an die Vorlage einer zu Lebzeiten von den StudienteilnehmerInnen eingeholten Einwilligung gebunden. Die Einwilligung muss sich dabei explizit auf (1) das Einverständnis zur Todesursachenrecherche und (2) die ärztliche Schweigepflichtentbindung zu diesem Zweck beziehen.

Abgesehen von Nordrhein-Westfalen, wo keine Todesursachenrecherche möglich ist, kommt es in Berlin und in Rheinland-Pfalz aufgrund der verkürzten Auf- 


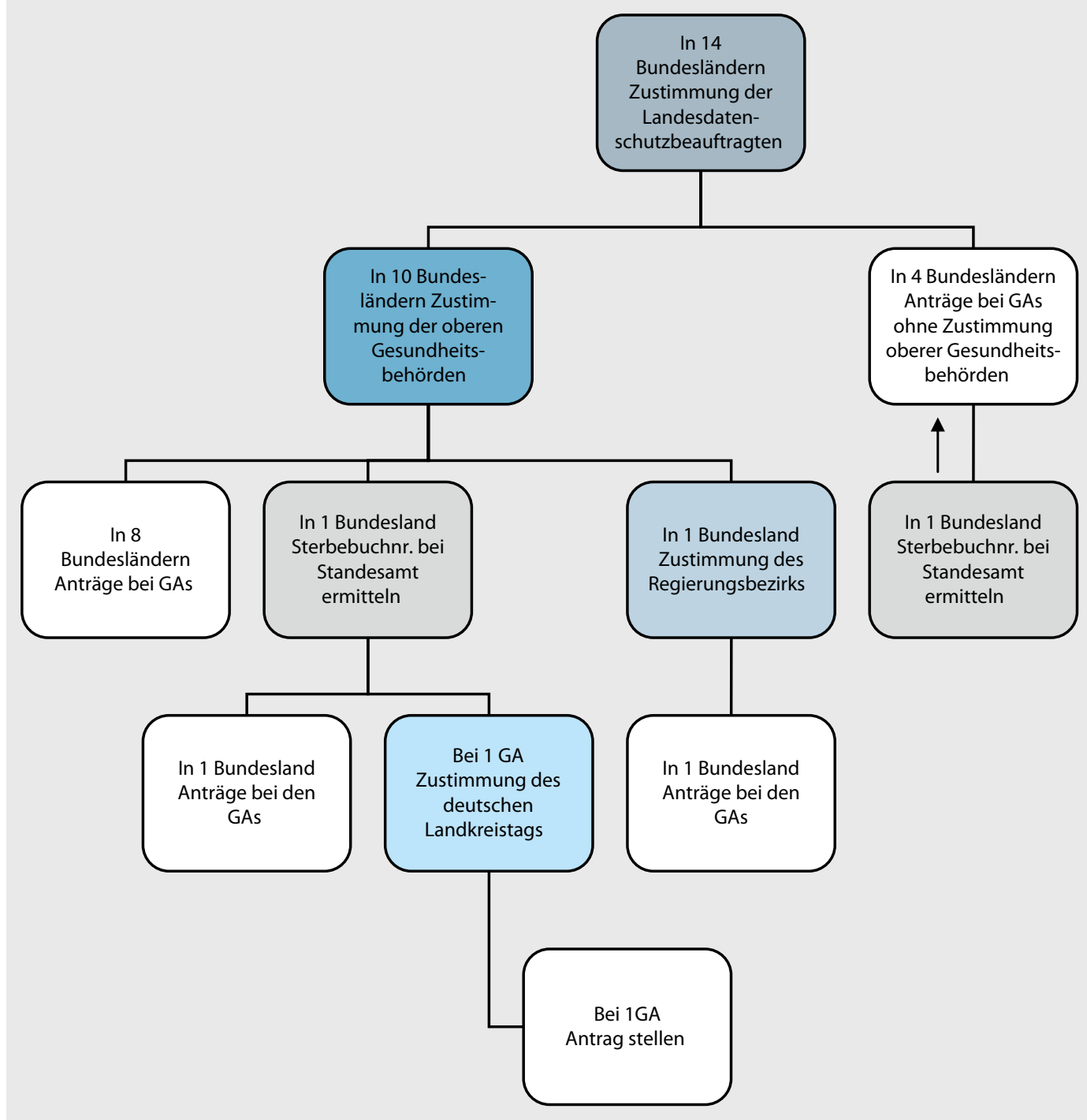

Abb. $1<$ Zugangswege zur retrospektiven Recherche von Todesursachen für alle Bundesländer (außer Berlin und Nordrhein-Westfalen). Stand April 2011. GA Gesundheitsamt bewahrungszeiten der Leichenschauscheine zu Datenverlusten.

\section{Fazit}

Im vorliegenden Pilotprojekt können Abläufe zur Recherche von Todesursachen anhand einer Stichprobe verstorbener Studienteilnehmerlnnen einer bundesweiten Kohortenstudie recherchiert und etabliert werden. Da kein nationales Mortalitätsregister existiert, ist eine Todesursachenrecherche in allen 16 Bundesländern notwendig. Über die beschriebenen Abläufe können in 13 von 16 Bundesländern Todesursachen für sämtliche ausgewählte Fälle und in zwei Bundesländern für einen Teil der ausgewählten Fälle recherchiert werden. Zu beachten ist, dass aktuelle Datenschutzbestimmungen die zu Lebzeiten eingeholte schriftliche Einwilligung der Studienteilnehmerlnnen voraussetzen, um Informationen zur Todesursache bei den Datenhaltern (in der Regel die regionalen Gesundheitsämter) zu erhalten. Darüber hinaus kann in fast allen Bundesländern (außer in Nordrhein-Westfalen) in begründeten Fällen eine Sondergenehmigung des Landesdatenschutzbeauftragten zur Recherche von Todesursachen bei verstorbenen Kohortenmitgliedern, auch ohne Einwilligungserklärung der Verstorbenen, eingeholt werden. Nach Erwerb dieser Sondergenehmigung kann eine Zustimmung bei den obersten Landesgesundheitsbehörden (soweit diese notwendig ist) beantragt werden. Der Aus- fall von Nordrhein-Westfalen ist für die retrospektive Todesursachenrecherche als sehr problematisch anzusehen, da bei lange bestehenden bundesweiten Kohorten wie dem BGS98 durch diesen Ausfall ein hoher Datenverlust in Bezug auf die Todesursachenrecherche $(16,6 \%$ der bislang registrierten Sterbefälle) zu erwarten ist. Weitere Datenverluste sind durch Verjährung der Aufbewahrungsfrist in Rheinland-Pfalz und Berlin zu erwarten, sodass hier besonders zeitnah recherchiert werden muss.

Das Erheben von Todesursachen ist unter den gegebenen Bedingungen mit hohem Zeit- und Personalaufwand verbunden. Zudem muss damit gerechnet werden, dass einmal etablierte Abläufe fortlaufend angepasst werden müssen, da sich die länderspezifischen Rah- 
menbedingungen ändern können. Bei allem Interesse und der Unterstützung, die das Pilotprojekt zur Todesursachenrecherche bundesweit von den beteiligten Behörden, Ämtern und Institutionen erfährt, ist die Etablierung eines Nationalen Mortalitätsregisters daher wünschenswert. Dies würde die Forschungsarbeit wesentlich erleichtern und dazu beitragen, dringend benötigte Daten für die Gesundheitspolitik zeitnah zur Verfügung stellen zu können. In diesem Zusammenhang ist die Initiative des Rates für Wirtschafts- und Sozialdaten (RatSWD) zu nennen, die sich nachdrücklich für den Aufbau eines nationalen Mortalitätsregisters in Deutschland ausspricht [6]. Solange jedoch in Deutschland kein bundesweites Mortalitätsregister existiert, ist es sinnvoll, Todesursachenrecherchen, unter den gegebenen Bedingungen in Zusammenarbeit mit den zuständigen Bundes- und Landesbeauftragten für Datenschutz und obersten Landesgesundheitsbehörden zu optimieren.

An dieser Stelle ist auch auf das grundsätzliche Problem der Datenqualität aus den Todesbescheinigungen hinzuweisen, dessen sich die Autoren zwar bewusst sind, auf das aber an dieser Stelle nicht eingegangen werden kann.

\section{Korrespondenzadresse}

\section{I.K. Wolf}

Robert Koch-Institut FG 22

General-Pape-Str. 62-64/66, 12101 Berlin Wolfi@rki.de

Danksagung. Wir bedanken uns bei allen Landesdatenschutzbeauftragten und dem Bundesdatenschutzbeauftragen für ihre Unterstützung. Unser Dank gilt den Gesundheitsministerien der Länder, dem Gemeinsamen Krebsregister Berlin-Brandenburg, dem Amt für Statistik Berlin, den mitwirkenden Ärzten Berlins, dem Deutschen Landkreistag, den Krankenhäusern und Standesämtern und allen Gesundheitsämtern, die uns bundesweit unterstützt haben.

Finanzierung/Laufzeit des Pilotprojekts. Das Mortalitäts-Follow-Up wurde vom Bundesministerium für Gesundheit (BMG) finanziert und vom 17.01.201115.09.2011 durchgeführt.

Interessenkonflikt. Der korrespondierende Autor gibt an, dass kein Interessenkonflikt besteht.

\section{Literatur}

1. Stafford N (2010) Germany moves closer to establishing a national mortality register. BMJ 340:c2517

2. Giersiepen K, Brunings-Kuppe C, Lehmann C (2004) The Bremen mortality index. Bundesgesundheitsbl Gesundheitsforsch Gesundheitsschutz 47(5):451-456

3. Landesdatenschutzgesetze. http://www.bfdi. bund.de/DE/AnschriftenUndLinks/Landesdatenschutzbeauftragte/AnschriftenLandesdatenschutzbeauftragte.html

4. Bundesdatenschutzgesetz. http://www.bfd.bund. de/

5. Metschke R, Wellbrock R (2002) Berliner Beauftragter für Datenschutz und Informationsfreiheit. Hessischer Datenschutzbeauftragter. Datenschutz in Wissenschaft und Forschung. Hrsg.: Berliner Beauftragter für Datenschutz und Informationsfreiheit

6. RatSWD (2010) Ein Nationales Mortalitätsregister für Deutschland. Bericht der Arbeitsgruppe und Empfehlung des Rates für Sozial und Wirtschaftsdaten (RatSWD). http://www.ratswd.de/download/publikationen_rat/Bericht_Empfehlung_Mortalitaetsregister.pdf 